\title{
Influence of gradients in the elastic anisotropy on the reliability of residual stresses determined by the hole drilling method
}

\author{
Jörn Niehuesbernd ${ }^{1, a}$, Enrico Bruder ${ }^{1, b}$, Clemens Müller $^{1, c}$ \\ ${ }^{1}$ Division of Physical Metallurgy, Materials Science Department, Darmstadt University of \\ Technology, Alarich-Weiss Straße 2, 64287 Darmstadt, Germany \\ j.niehuesbernd@phm.tu-darmstadt.de, be.bruder@phm.tu-darmstadt.de, \\ cc.mueller@phm.tu-darmstadt.de
}

Keywords: Hole drilling method, texture, elastic anisotropy, linear flow splitting

\begin{abstract}
Metal forming processes often involve large strain gradients which results in heterogeneous deformation and consequently residual stresses. Furthermore the strain gradients also generate variations in the deformation texture and related properties. For materials with a significant crystallographic elastic anisotropy such as ferritic steel, these textures may have a substantial effect on the reliability of the determination of residual stresses. In the present investigation this influence is examined for the hole drilling method by a combination of experiments and finite element simulations.
\end{abstract}

\section{Introduction}

The development of modern forming processes in combination with the adaption of material properties has led to procedures which introduce large strains and in most cases significant strain gradients in the material, which generally cause residual stresses. Since plastic deformation also leads to the development of crystallographic textures, the influence of these textures on determined residual stress distributions should be considered. Furthermore, for processes which introduce large strain gradients and spatially changing deformation modes, gradients in crystallographic textures and related elastic properties can occur. A process which involves these complex forming conditions is linear flow splitting (LFS), which produces bifurcations by subjecting the edges of a sheet to severe plastic deformation [1]. Obtuse angled splitting rolls drive into the edges of the sheet while supporting rolls on both sides of the sheet hinder buckling and plastic deformation in thickness direction (fig. 1a). Through several steps flanges are being produced, leading to profiles with a double-Y shape. Owing to the severe strains and the complex forming conditions high residual stresses develop within the flanges of the profiles [2].

The material flow during LFS is perpendicular to the feed direction. Therefore the direction from the splitting center to the flange tip can be treated as the rolling direction (RD), analog to regular sheet rolling (fig. 1b). Consequently the feed direction corresponds to the Transverse direction (TD) and the in-thickness direction to the normal direction (ND).

In consequence of the heterogeneous material flow and steep strain gradients in the process zone, strong microstructure and yield strength gradients in flange thickness direction develop during the process [3]. Earlier investigations on HSLA steels also have shown the occurrence of strong crystallographic textures, leading to a significant elastic anisotropy with deviations in Young's modulus of $15 \%$ compared to the isotropic mean [4, 5]. Since the elastic properties of a polycrystalline material with a substantial crystallographic elastic anisotropy are linked to its texture, it is advantageous to determine the local elastic properties directly from the grain orientation distribution. A calculation method which has proven to be a suitable approximation is the geometric mean $[5,6,7]$. In this case the single crystal stiffness tensor $(C)$ of the examined material is rotated according to measured grain orientations using rotation matrices $(T)$. Grain 
orientation data from electron backscatter diffraction measurements (EBSD) can directly be used in the calculations. For the geometric mean the natural logarithm of the rotated stiffness tensors has to be calculated before averaging over all measured points $(N)$ :

$$
C_{\text {geom }}=\exp \left(\frac{1}{N} \sum_{i}^{N} \ln \left(\left[T_{i}\right]^{T}[C]\left[T_{i}\right]\right)\right)
$$

Based on the observation of significant texture gradients in HSLA steel profiles produced by LFS [5], it is reasonable to assume that texture gradients develop during LFS of other ferritic steels as well. Furthermore these texture gradients might also lead to gradients in the elastic anisotropy. A series of investigations already discussed the determination of residual stress measurements on orthotropic and anisotropic materials using the hole drilling method $[8,9]$, but the influence of gradients in the elastic properties has not been investigated yet.

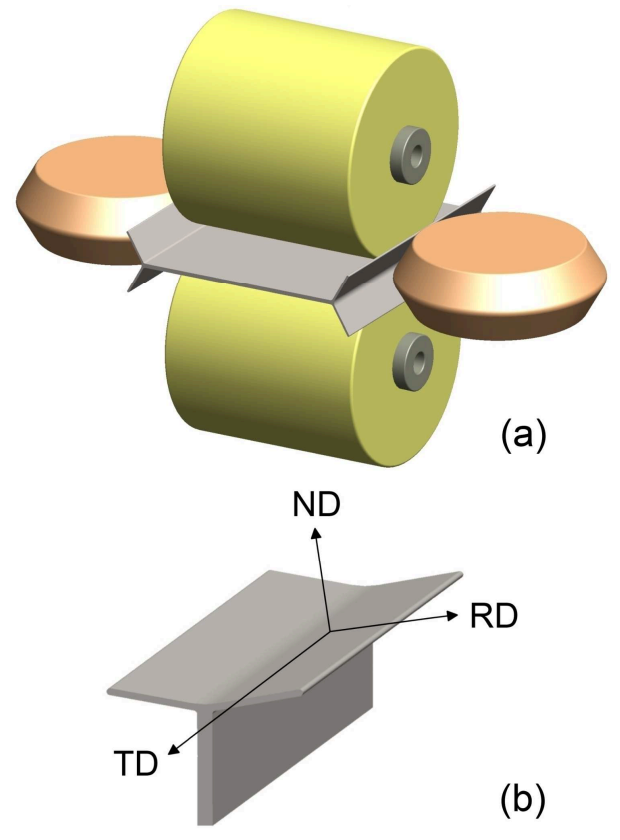

Fig. 1: a) Function principle of linear flow splitting, b) defined coordinate system

\section{Experimental}

The investigations were carried out on the ferritic stainless steel X6Cr17 (AISI 430/1.4016) with the nominal composition (wt\%): $16-18 \mathrm{Cr}, 0.08 \mathrm{C}, 1.0 \mathrm{Mn}, 1.0 \mathrm{Si}, 0.04 \mathrm{P}, 0.01 \mathrm{~S}$. The microstructure consists of ferrite grains with chromium carbide precipitations distributed homogeneously within the grains. The thickness and width of the initial sheet were 2 and $90 \mathrm{~mm}$ respectively. The doubleY-profiles with flange lengths of approximately $9 \mathrm{~mm}$ and flange thicknesses of about $1.1 \mathrm{~mm}$ were produced by continuous linear flow splitting. Hardness measurements and texture investigations were carried out on cross sections of the produced profiles. Standard metallographic grinding and polishing techniques followed by an additional polishing step using an aqueous suspension of $0.02 \mu \mathrm{m} \mathrm{Al} \mathrm{O}_{3} \mathrm{O}_{3}$ were applied to obtain a smooth and deformation free surface. Hardness profiles were measured by the Vickers method with a load of $50 \mathrm{~g}$ at half the distance between the splitting center and the flange tip with progressively growing distance to the flange top surface. Due to the steep hardness gradient in the flange thickness direction tensile tests are not suitable to gain information about the local yield strength along the gradient. Therefore an experimental relation between Vickers hardness and yield strength was derived from tensile tests of the as received sheet material. Since the maximum degree of deformation for the tensile tests was about 0.15 and the estimated degree of deformation in the highly strained regions of the flanges is $>>1$, the linear correlation was extrapolated by assuming a constant strain hardening exponent. These considerations led to the following equation:

$\sigma_{y s}=3.13 \cdot H V 0.05-180$

The hardness data in flange thickness direction was used to calculate the local yield strength at the respective measurement position. Earlier investigations on HSLA steels have proven this method to be a suitable approximation [10].

Grain orientation measurements using EBSD were carried out at the corresponding positions of the hardness measurements in order to obtain textures and elastic properties. Textures were represented in terms of orientation distribution functions (ODF), calculated using harmonic series expansion with a series rank of 24 , a Gaussian half width of $5^{\circ}$ and the assumption of orthotropic symmetry. 
Until now no experimental data exist on the single crystal elastic constants of ferrite steels with a Chromium content of $17 \%$. However, ab initio calculations showed that $\mathrm{Cr}$ only has a minor impact on the elastic properties of ferrite iron [11]. Therefore the single crystal elastic constants of pure iron with $\mathrm{C}_{11}=230.1 \mathrm{GPa}, \mathrm{C}_{12}=134.6 \mathrm{GPa}$ and $\mathrm{C}_{44}=116.6 \mathrm{GPa}$ were used to calculate the local elastic anisotropy.

Residual stress measurements were performed at the flange top surface, at half the distance between the splitting center and the flange tip, using an MTS3000 system. Drills with a diameter of $0.8 \mathrm{~mm}$ and strain gage rosettes with a grid centerline diameter of $2.56 \mathrm{~mm}$ (Micro-Measurements EA-06031RE-120) were used to drill holes incrementally to a depth of $0.8 \mathrm{~mm}$ with an increment size of $25 \mu \mathrm{m}$. The orthogonal strain gages of the rosettes were aligned along RD and TD respectively.

Finite element simulations were carried out using Abaqus (implicit). Only a quarter of the drilling hole surroundings was modeled in order to reduce computation time. The modeled geometry covered the whole specimen thickness in order to rule out thickness depending deviations. Meshing was done using hexahedral elements (C3D20R). Near the hole the in-plane seed distance was chosen as $0.1 \mathrm{~mm}$. With increasing distance to the hole the seed distance was increased up to $2 \mathrm{~mm}$. In thickness direction the sheet was partitioned into $50 \mu \mathrm{m}$ thick layers. Each layer was assigned a specific yield strength and stiffness tensor, corresponding to the experimental data. Data in between measured points was obtained by linear interpolation. The residual stress distribution was introduced by assigning each layer initial stress values in RD and TD. In order to obtain depth dependent stain data, slices with a thickness of $50 \mu \mathrm{m}$ and a diameter of $1.1 \mathrm{~mm}$ (corresponding to the actual hole diameter) were removed in a series of steps to a final depth of $0.8 \mathrm{~mm}$. The surface strains were acquired in terms of point data on parallel lines, corresponding to the actual grid lines of the strain gage rosette. The resulting depth dependent strain behavior was compared to the measured one and the initially assigned stresses were varied iteratively until the measured and the simulated strains showed differences of less than $0.5 \%$. The same procedure was carried out for the case of an isotropic elasticity to assess the impact of anisotropy on residual stress values determined by the hole drilling method

\section{Results and discussion}

The examined flanges exhibit a hardness gradient with a maximum value of about $370 \mathrm{HV} 0.05$ at $25 \mu \mathrm{m}$ beneath the split surface, dropping to around $290 \mathrm{HV} 0.05$ at the bottom surface (fig. 2). The heterogeneous forming conditions also become apparent by examining local textures within the gradient direction. The textures determined by EBSD measurements are represented in terms of ODFs for the respective measurement positions (fig. 3). The severity of the textures, measured by the mean square value of the orientation distribution i.e. the texture index, drops from 5.8 at $25 \mu \mathrm{m}$ beneath the split surface to a value of 1.5 at the bottom surface. Furthermore the rolling textures with partial $\alpha$-fibers (red/vertical) and $\gamma$-fibers (blue/horizontal), which can be observed near the split surface, vanish with increasing depth. In addition, with increasing distance to the split surface the fiber with $\{100\} \|$ ND starts to gain intensity. At the bottom surface of the flange moderate intensities can be observed for the cube orientation $(\{001\}<010>)$ and Goss orientation $(\{110\}<001>)$, the latter one being an indicator for shear components in deformation.

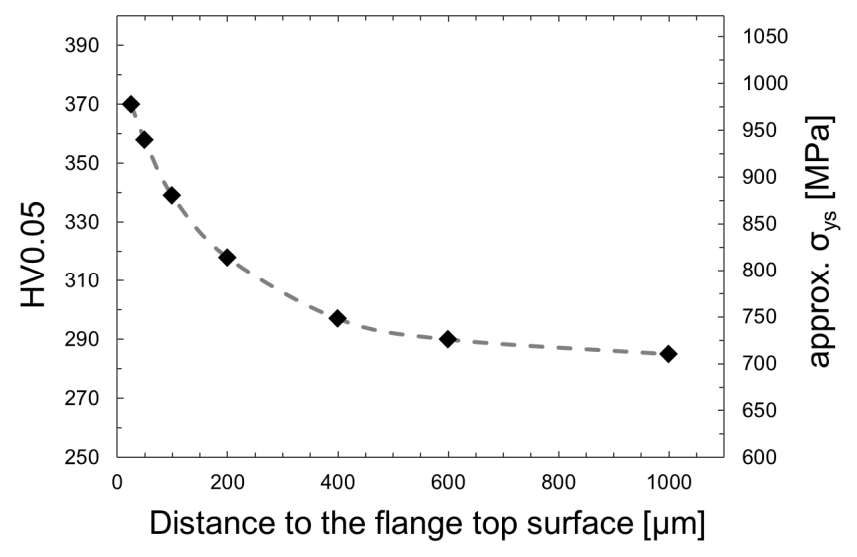

Fig. 2: Hardness and yield stress gradient in thickness direction of the flanges of $\mathrm{X} 6 \mathrm{Cr} 17$ split profiles 

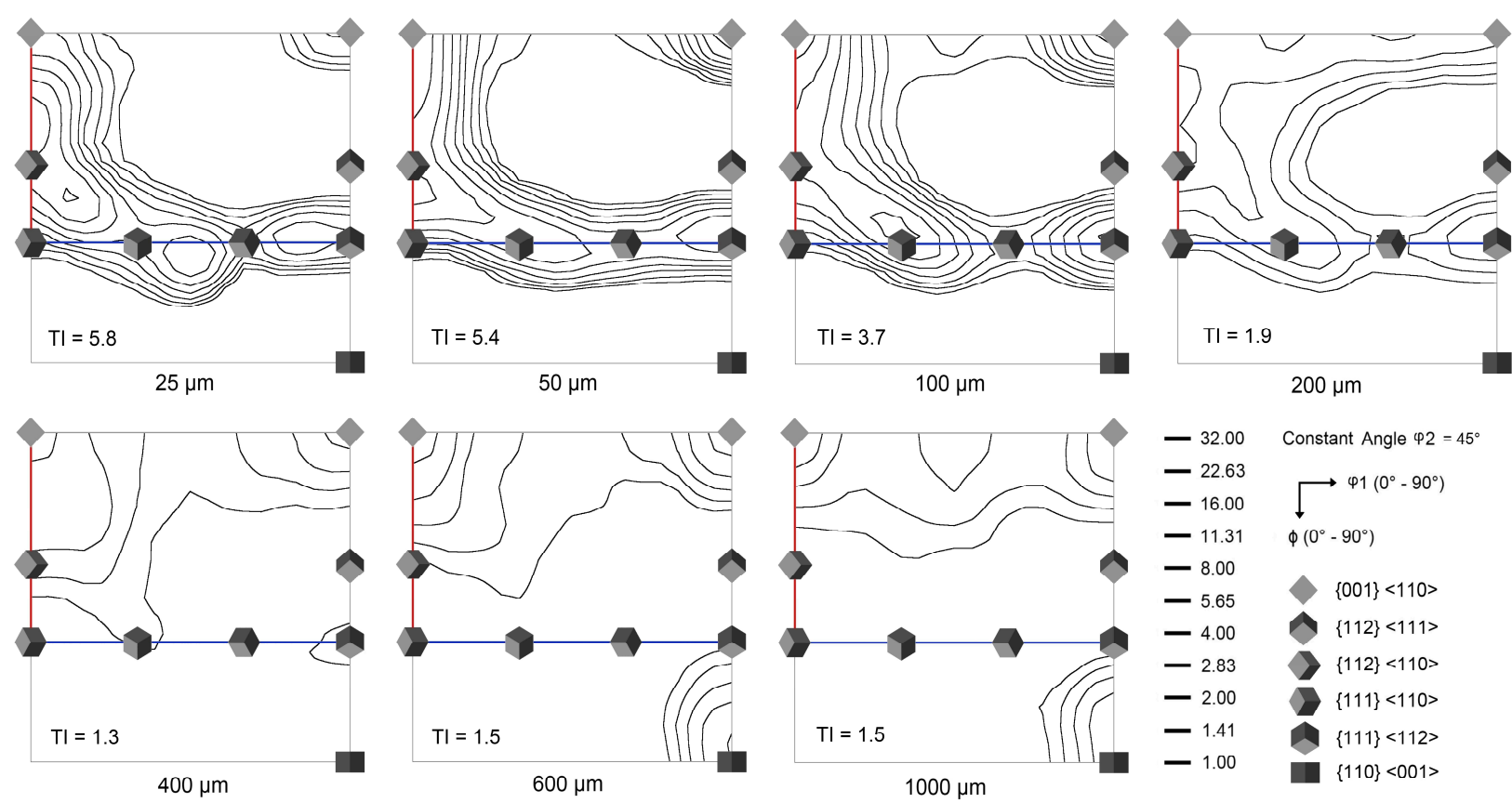

Fig. 3: Orientation distribution functions of several measurement positions. Only sections of the Euler space with a constant angle $\phi 2=45^{\circ}$ are shown.

The determined Young's modulus distributions for the respective measurement positions on the one hand reveal a strong elastic anisotropy and on the other hand also show significant changes in the character of the anisotropy with respect to the measurement depth (fig. 4). Close to the split surface the anisotropy is defined by the rolling texture i.e. by the $\alpha-$ and $\gamma$-fibers. Therefore maximum values can be found in transverse direction (TD) and in rolling direction (RD), while minimum values lie under $45^{\circ}$ between RD and TD and between TD and ND. The maximum in transverse direction is of special interest, since along the length of the profile one of the principal stress directions is expected. The maximum value in 25 $\mu \mathrm{m}$ beneath the split surface is 244 $\mathrm{GPa}$, which is more than $16 \%$ higher than the isotropic mean of $210 \mathrm{GPa}$. With increasing distance to the split surface the Young's modulus in TD approaches the isotropic mean. Other directions with major changes are RD and ND. The relative change in Young's modulus in RD between top and bottom surface is approximately

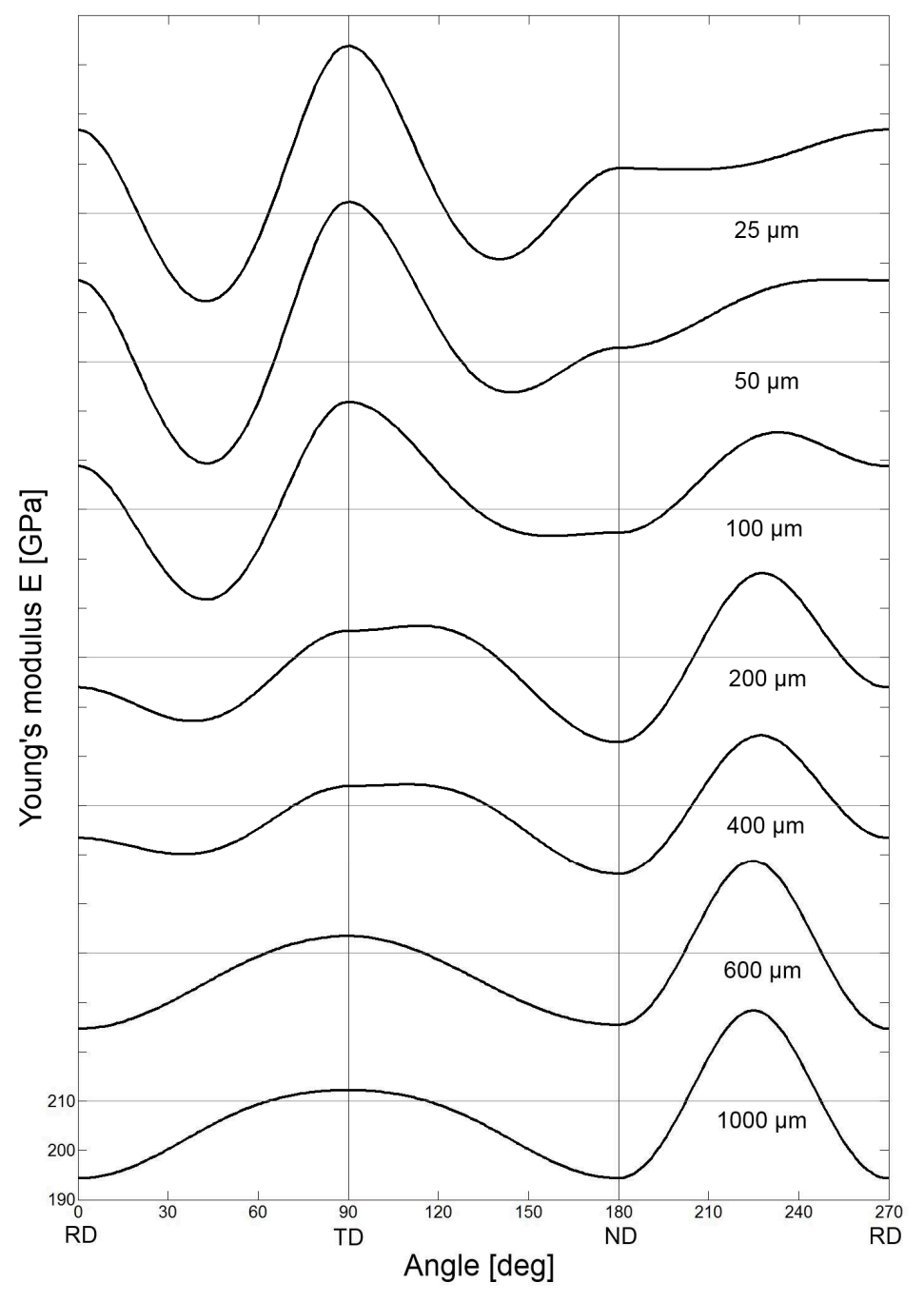

Fig. 4: Young's modulus behavior between the main sample directions RD, TD and ND for several measurement positions 
$14 \%$, which is nearly as high as the change in TD.

The results of the FE-simulations of the drilling process reveal very high residual stress levels in feed direction (TD) of the split profiles (fig. 5). Nearly $800 \mathrm{MPa}$ of tensile stress in a depth between $0.1 \mathrm{~mm}$ and $0.2 \mathrm{~mm}$ can be observed for the isotropic as well as for the anisotropic (orthotropic) case. During the drilling process plastic deformation occurs at the vicinity of the hole, especially in the upper $0.2 \mathrm{~mm}$ (fig. 6). The main plastic strains localize at the rim of the hole, perpendicular to the direction of the high stress values and at the edge between the rim and the bottom of the hole. Even though the plastic strains do not reach the area underneath the strain gages, the nonlinear relaxation superimposes the elastic one. As a result the recorded surface strains are mainly defined by plastic deformation, which is the reason for the negligible difference in the determined stresses between the isotropic and the orthotropic case within the first $0.2 \mathrm{~mm}$ beneath the split surface. The determined stresses in subjacent layers also are of considerable interest. In a depth between $0.3 \mathrm{~mm}$ and $0.4 \mathrm{~mm}$ the

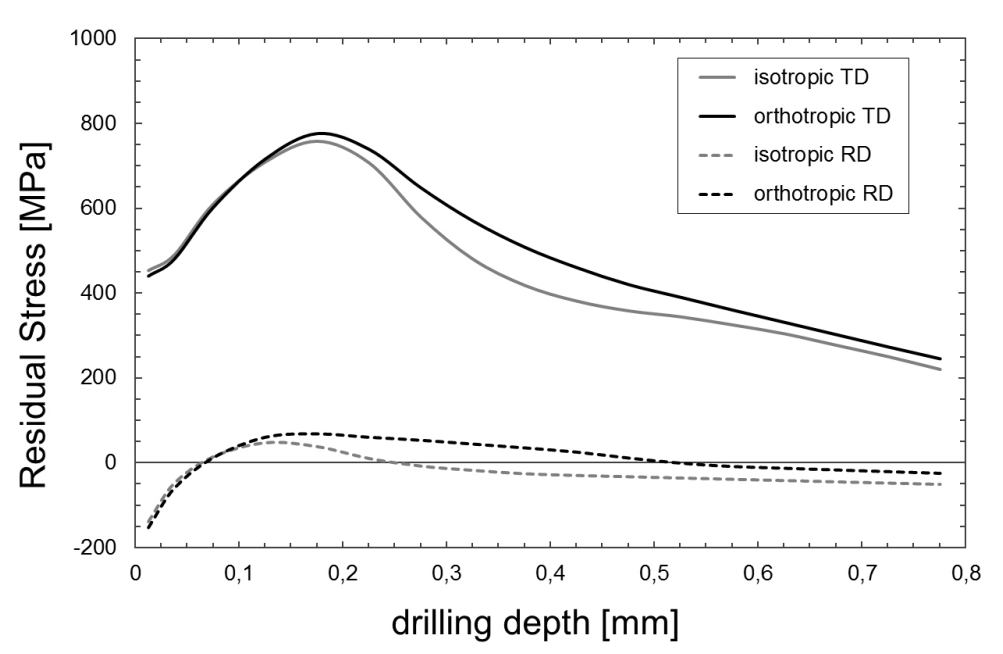

Fig. 5: Determined residual stresses for the isotropic and the orthotropic FE-model in TD and RD

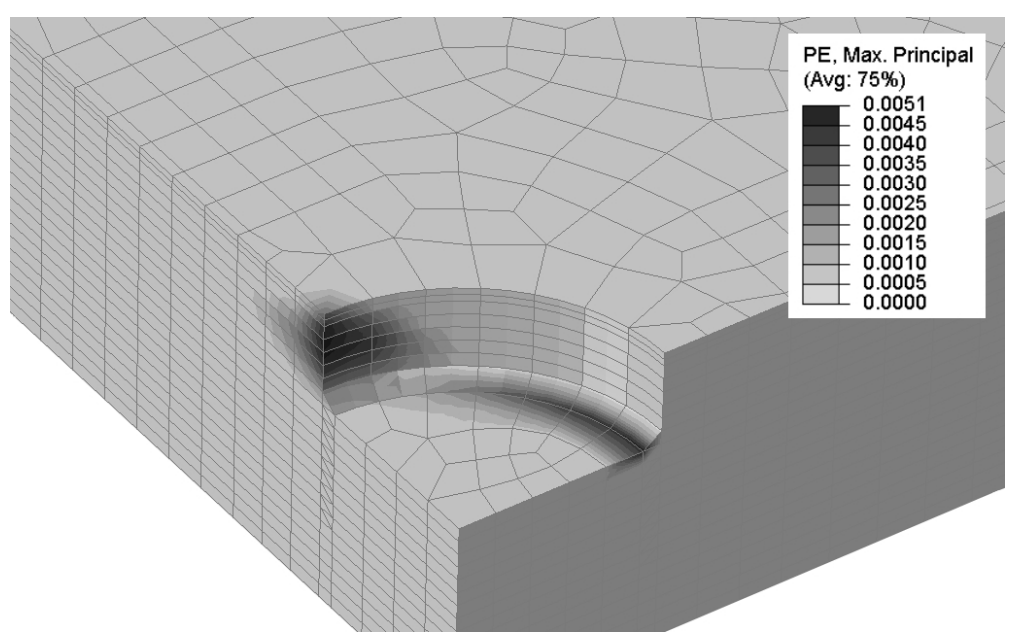

Fig. 6: Plastic strains at the rim and the bottom of the drilled hole stress levels for the orthotropic model are about $20 \%$ higher than the one for the isotropic model. In this depth the difference between the Young's moduli of the two models is only $3 \%$. It is reasonable to assume, that the stiffer upper layers in the orthotropic model diminish the surface relaxation caused by the removal of a layer with a lower stiffness in the examined direction. This means that for the isotropic model the residual stresses in lower layers are highly underestimated. According to this behavior the assumption can be derived that for the hole drilling method anisotropic elastic properties not only influence the determination of residual stresses in layers where anisotropy is present, but also in subjacent layers which might have isotropic properties.

\section{Summary and conclusion}

Crystallographic textures which lead to anisotropic elastic material properties have a significant impact on the stress levels determined with the hole drilling method. In this method the elastic surface relaxations caused by the removal of material in lower layers are strongly dependent on the relaxations in overlying layers. Therefore the elastic properties of upper layers have a strong influence on the calculated residual stresses in subjacent layers. In consequence, the elastic anisotropy, especially in near surface layers, has to be taken into account, in order to determine 
exact residual stress distributions. However, for very high residual stress levels the surface strains are dominated by plastic deformation in the vicinity of the drilled hole, making the influence of elastic anisotropy negligible. In this case exact residual stress values can only be determined with an accurate knowledge of the materials yield strength and the plastic behavior.

\section{References}

[1] P. Groche, D. Vucic, M. Jöckel, Basics of linear flow splitting, J. Mater. Process. Technol. 183 (2007) 249-255.

[2] V. Landersheim, B. Eigenmann, C. el Dsoki, T. Bruder, C. M. Sonsino, H. Hanselka, Analyse der Wirkung von Kerben, Mittel- und Eigenspannungen auf die Schwingfestigkeit des hochumgeformten Werkstoffbereichs von Spaltprofilen, Materialwiss. Werkst. 40 (2009) 663-675.

[3] T. Bohn, E. Bruder, C. Müller, Formation of ultrafine-grained microstructure in HSLA steel profiles by linear flow splitting, J. Mater. Sci. 43 (2008) 7307-7312.

[4] E. Bruder, The effect of deformation texture on the thermal stability of UFG HSLA steel, J. Mater. Sci. 47 (2012) 7751-7758

[5] J. Niehuesbernd, C. Müller, W. Pantleon, E. Bruder, Quantification of local and global elastic anisotropy in ultrafine grained gradient microstructures produced by linear flow splitting, Mat. Sci. Eng. A 560 (2013) 273-277.

[6] S. Matthies, M. Humbert, the realization of the concept of a geometric mean for calculating physical constants of polycrystalline materials, Phys. Status Solidi B 177 (1993) K47-K50.

[7] S. Matthies, M. Humbert, On the Principle of a Geometric Mean of Even-Rank Symmetric Tensors for Textured Polycrystals, J. Appl. Crystallogr. 28 (1995) 254-266.

[8] T. Schwarz, Beitrag zur Eigenspannungsermittlung an isotropen, anisotropen sowie inhomogenen, schichtweise aufgebauten Werkstoffen mittels Bohrlochmethode und Ringkernverfahren, Staatliche Materialprüfanstalt (MPA) Universität Stuttgart 1996.

[9] G. S. Schajer, L. Yang, Residual-stress measurement in orthotropic materials using the holedrilling method, Exp. Mech. 34 (1994) 324-333.

[10] C. Müller, T. Bohn, E. Bruder, T. Bruder, V. Landersheim, C. el Dsoki, P. Groche, D. Veleva, Severe plastiv deformation by linear flow splitting, Mat. Wiss. u. Werkstofftech. 38 (2007) 842854.

[11] H. Zhang, B. Johansson, L. Vitos, Ab inito calculations of elastic properties of bec Fe-Mg and Fe-Cr random alloys, Phys. Rev. B 79 (2009) 224201-1-224201-10 